\title{
Innovación e integración productiva en un sistema bovino con doble propósito del noroeste de México
}

\section{Innovation and Productive Integration in a Dual-purpose Bovine System in Northwest Mexico}

\author{
Alma Delia Santiago Solano* (iD https://orcid.org/0000-0002-5084-7272 \\ Lorenia Velázquez Contreras ${ }^{\star *}$ (D) https://orcid.org/0000-0002-1367-1001 \\ Jesús Mario Moreno Dena*** iD https://orcid.org/0000-0002-5644-305X \\ Vidal Salazar Solano ${ }^{\star \star * \star}$ (D) https://orcid.org/0000-0002-9906-9168
}

\begin{abstract}
Resumen
El objetivo del presente estudio es caracterizar los procesos de innovación tecnológica y organizacional que contribuyeron a la integración del sistema productivo bovino con doble propósito en Suaqui Grande, Sonora, México. Mediante la aplicación de entrevistas semiestructuradas a miembros de una muestra de I2 cooperativas se construyó una representación cronológica-narrativa de los procesos de innovación. Los resultados muestran cómo la reconfiguración del esquema ejidal en estas empresas familiares posibilitó la integración del sistema productivo. Así mismo, se encontró que el empoderamiento de la Unión de Los Soakis en los eslabones de la cadena de valor se sustentó en la inducción de innovaciones en su organización, equipamiento e infraestructura, entre otros rubros importantes, pero en especial en una visión compartida de desarrollo. Se concluye que la gestión de las innovaciones tecnológica y organizacional conformó el soporte de la integración productiva de las comunidades participantes entre 2010 y 2015. Una limitación fue que el tiempo transcurrido entre la adopción de innovaciones y la recabación de información no permitió un análisis a fondo de los efectos socioeconómicos en los productores y en el territorio. Palabras clave: innovación; ganadería; producción primaria; gobernanza; integración productiva.
\end{abstract}

\begin{abstract}
This paper aims to characterize the technological and organizational innovation processes that contributed to the integration of the dual-purpose livestock production system in Suaqui Grande, Sonora, Mexico. A chronological narrative representation of innovation processes was constructed through the application of semi-structured interviews to members of a sample of 12 cooperatives. The results show how the reconfiguration of the ejido scheme in these family businesses made the integration of the productive system possible. In addition, it was found that the empowerment of Unión de Los Soakis in the value chain links was based on the induction of innovations in their organization, equipment, and infrastructure, among other transcendental areas, but above all, on a shared vision of development. It is concluded that the management of technological and organizational innovations constituted the support for the productive integration of the participating communities between 2010 and 2015. A limitation was that the time elapsed between the adoption of innovations and the collection of information did not allow an in-depth analysis of producers and territory socioeconomic effects.

Keywords: innovation; livestock; primary production; governance; productive integration.
\end{abstract}

Cómo citar: Santiago Solano, A. D., Velázquez Contreras, L., Moreno Dena, J. M., y Salazar Solano,V. (2020). Innovación e integración productiva en un sistema bovino con doble propósito del noroeste de México. región y sociedad, 32, el 372. doi: I0.22 I98/rys2020/32/I372

*El Colegio de Sonora. Avenida Obregón Núm. 54, Col. Centro, C. P. 83000, Hermosillo, Sonora, México. Correo electrónico: aldesan62@gmail.com

**El Colegio de Sonora. Avenida Obregón Núm. 54, Col. Centro, C. P. 83000. Hermosillo, Sonora, México. Correo electrónico: Ivelaz@colson.edu.mx

*** Centro de Investigación en Alimentación y Desarrollo. Carretera Gustavo Enrique Astiazarán Rosas Núm. 46, Col. La Victoria, C. P. 83304, Hermosillo, Sonora, México.Correo electrónico: jesus.moreno@ciad.mx

**** Autor para correspondencia. Centro de Investigación en Alimentación y Desarrollo. Carretera Gustavo Enrique Astiazarán Rosas Núm. 46, Col. La Victoria, C. P. 83304, Hermosillo, Sonora, México. Correo electrónico: vidal@ciad.mx 


\section{Introducción}

En un entorno de limitaciones para resolver problemas cada vez más complejos, el Estado tiende a ceder la toma de decisiones sobre los lineamientos rectores de la producción alimentaria a las corporaciones que la dominan (Torfing y Triantafillou, 2016). En tal escenario, los pequeños productores implementan innovaciones organizativas y tecnológicas para adaptarse a los condicionamientos que emergen de los mercados globalizados.

En el estado de Sonora, localizado en el noroeste de México, destacan las comunidades ganaderas del municipio de Suaqui Grande por su integración productiva y dinámica de crecimiento en un ambiente económico global adverso. Estas comunidades constituyeron la Unión de Sociedades del Centro Los Soakis, S. C. de R. L. de C. V. (Los Soakis), como parte de una estrategia de posicionamiento de sus unidades de negocios en los eslabones de la cadena de valor. Al estallar la crisis financiera, energética y ambiental que a escala global expuso la fragilidad de los sistemas agroalimentarios locales en 2008, Los Soakis contaban con fortalezas estructurales para sortear los efectos negativos que impactaron al resto de actores de la ganadería regional, con quienes compartían características socioeconómicas. El objetivo de este trabajo es caracterizar las innovaciones tecnológicas y organizativas inducidas como soporte de la integración económica del sistema productivo bovino lechero de Los Soakis, desde los albores del siglo XXI.

El documento está estructurado en seis secciones adicionales a la presente nota introductoria. En la primera se aborda el marco referencial para el análisis de las prácticas de innovación tecnológica y organizativa de Los Soakis. La segunda sección resume la configuración de los contextos regional y socioeconómico que acompañan tales procesos. En la sección tercera, se precisan los procedimientos metodológicos que dieron soporte a la investigación. La cuarta muestra los resultados respecto al proceso de gestión para la innovación tecnológica. La quinta describe el proceso de conformación de la Unión de Sociedades del Centro Los Soakis y el proyecto estratégico implementado para enfrentar la crisis alimentaria global. En la última sección se presentan las conclusiones del análisis, destacando el rol de las innovaciones tecnológicas y organizativas como soporte de la integración económica de las comunidades Soakis estudiadas.

\section{Innovación, aprendizaje colectivo y las organizaciones}

La innovación en una empresa es "la introducción de un nuevo o significativamente mejorado producto (bien o servicio), de un proceso, de un nuevo método de comercialización, o de un nuevo método organizativo en las prácticas internas de la empresa, la organización del lugar de trabajo o las relaciones exteriores" (Organización para la Cooperación y el Desarrollo Económicos [OCDE], 2005, p. 56). En esta concepción, el grado de novedad del cambio es básico para considerar su carácter innovador. La innovación no necesariamente impli- 
ca una invención, sino que puede ser la identificación o ajuste de nuevas ideas y soluciones tomadas de otras experiencias.

Las innovaciones son componentes importantes de la competitividad de una empresa. Su implementación implica dinámicas de aprendizaje entre los actores y se insertan en las redes sociales. Desde la perspectiva de proximidad geográfica, la interacción favorece la circulación de conocimientos (Alburquerque, 2017). En un sistema productivo agroalimentario, un proceso de innovación suele resultar de la combinación de conocimientos tácitos -que se transmiten cara a cara-, de aprender-haciendo y de conocimientos codificados que se adquieren a través de capacitación (Boucher y Reyes-González, 2016).

La comprensión de las transformaciones concurrentes en un territorio requiere el análisis de las limitaciones que regulan las redes de intercambio y la evolución de la organización y las instituciones. Esas limitaciones se asumen como las restricciones autoimpuestas por el hombre para dar forma a la interacción humana y las organizaciones son reconocidas como "el grupo de individuos enlazados por alguna identidad común hacia ciertos objetivos" (North, 1993, p. 15).

Cárdenas-Bejarano, Gallardo-López, Núñez-Espinoza, Asiaín-Hoyos, Rodríguez-Chessani y Velásquez-Beltrán (2016) corroboran que la adopción de tecnología por productores de los estados de Veracruz y Tampico, México, incorporados en el modelo de Grupos Ganaderos de Validación y Transferencia de Tecnología, se asoció de manera positiva con el incremento del tamaño de la red, las interacciones sociales y la educación de los ganaderos, así como de forma inversa con la edad de éstos.

Rangel, Espinosa, Pablos-Heredero, Barba, Vélez, Rivas y García (2017), al evaluar la adopción de tecnologías y prácticas administrativas en el sistema de doble propósito del trópico mexicano, confirmaron que éste se reveló como limitado y variable, según el área tecnológica (alimentación, reproducción y manejo), el tamaño del rebaño y la zona ecológica. En general, las pequeñas explotaciones de doble propósito precisan mejorar su nivel tecnológico, ya sea por adopción o por la vía de la implementación de prácticas organizativas. Ello implica profundizar en el conocimiento científico del sistema, en la gestion y en el soporte técnico público-privado con perspectiva transversal y multidisciplinaria desde la evaluación ex-ante de los proyectos y tecnologías a implementar hasta el acceso al financiamiento.

En América Latina, la debilidad de la gestión pública obstaculiza el desarrollo rural. Sin embargo, la capacidad organizativa y la persistencia de instituciones locales permiten la resiliencia y la innovación. En los territorios cuyo desarrollo se finca en la producción láctea como insumo de la elaboración de queso (el cotija en la sierra Jalmich de México, el Turrialba en la ruta turística en Costa Rica, el Cajamarca en Perú y la quesería andina en Ecuador), la reputación e historia del territorio, el saber hacer y los actores con capacidad de organizarse son elementos fundamentales de la gobernanza y van creando un contexto propicio para la adopción de innovaciones, donde la ganadería emerge como un activo patrimonial en el medio rural (Torres y Sanz, 2007). Al enfrentar los problemas, se crean oportunidades y una variedad de actores públicos y 
particulares que interactúan en el logro de objetivos y que van construyendo procesos de gobernanza incidentes en la definición del territorio (Farinós, 2005; Kooiman y Jentoft, 2009).

\section{Contexto económico y territorial de los criadores de ganado de Suaqui Grande}

Las comunidades ganaderas de Suaqui Grande se insertan en la zona intermontañosa del estado de Sonora, en el Distrito de Desarrollo Rural 145 Mazatán (DDR 145) (véase figura 1). Con $889.3 \mathrm{~km}^{2}$, el territorio está conformado por 17 comunidades interconectadas por una red de $152 \mathrm{~km}$ de caminos.

Figura 1. El DDR-145 Mazatán

y el municipio de Suaqui Grande, Sonora, México

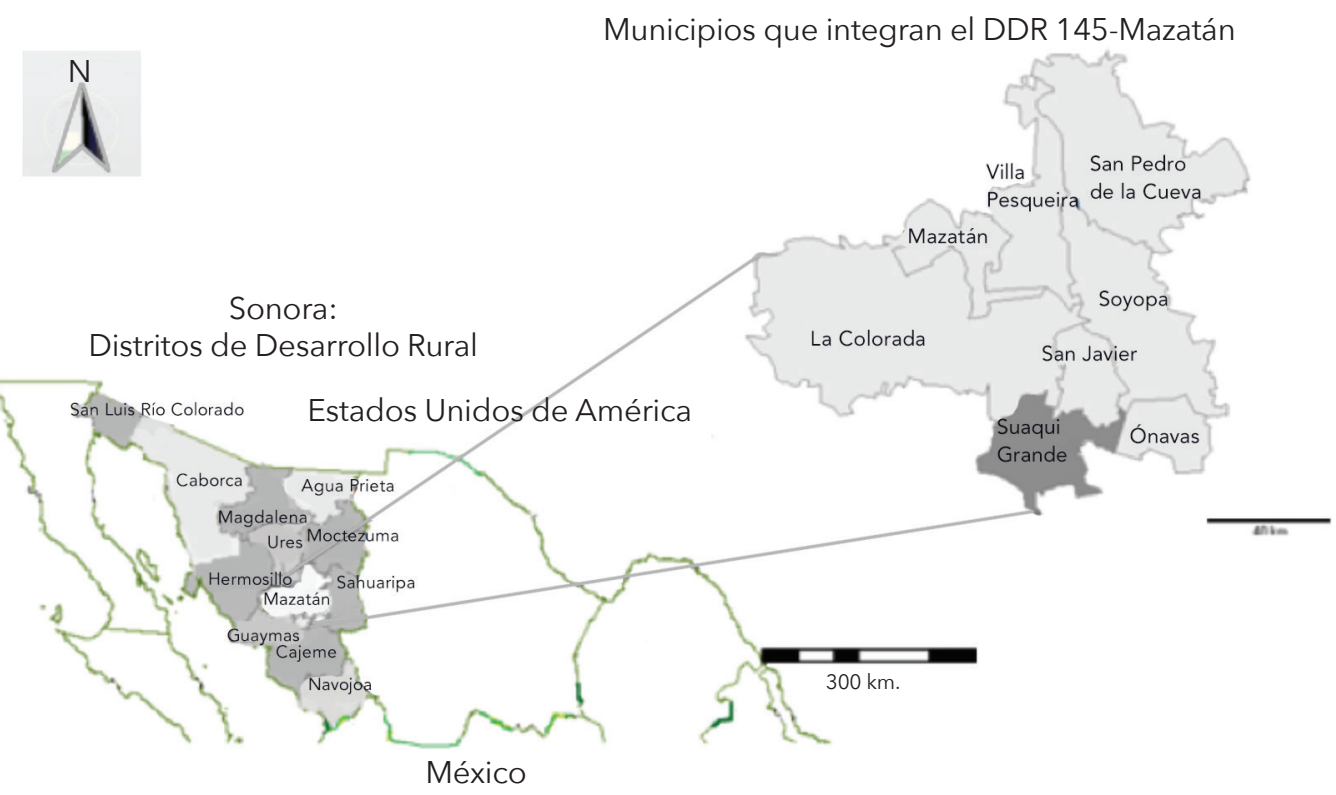

Fuente: elaboración propia con base en el Instituto Nacional de Estadística y Geografía (2015).

En este municipio de 1143 habitantes, 45.6\% pertenece a la población económicamente activa (Consejo Estatal de Población Sonora [COESPO], 2019). La estructura del empleo es liderada por las actividades primarias, que concentran $36.4 \%$ de la ocupación; le siguen el sector secundario con $31.7 \%$ y las actividades comerciales y de servicios, que concentran el restante $31.9 \%$ de ese segmento poblacional (COESPO, 2019).

México ha sido regionalizado en territorios con características ecológicas y socioeconómicas homogéneas con el objeto promover el desarrollo rural integral. Conforme a tales criterios, el estado de Sonora comprende doce distritos de desarrollo rural que ordenan el espacio de los 72 municipios que conforman su división política (Diario Oficial de la Federación [DOF], 1988). 
En la conformación de la estructura ocupacional sobresale el sistema de explotación bovino de doble propósito, caracterizado por una elevada eficiencia relativa, que influye en el patrón de acumulación regional. Los ingresos económicos de los actores de la cadena bovino-leche-carne son complementados con la elaboración de derivados lácteos, carne deshidratada, conservas y licor destilado de maguey (bacanora), entre otras actividades. El liderazgo del municipio en el aprovechamiento de las vocaciones productivas ganaderas del DDR 145 se construyó desde inicios del siglo XXI, cuando los pequeños criadores trascendieron de sus prácticas tradicionales al implementar un proyecto estratégico de integración económica, enfocado a promover la competitividad de sus unidades productivas.

Contexto económico y político del surgimiento de las comunidades Soakis

En México, la crisis ganadera se remonta al cambio de modelo económico en la década de 1980. El impulso al proceso de apertura comercial y la firma del tratado de libre comercio con Estados Unidos y Canadá favorecieron a los grandes productores de México (Rubio, 2013) y restaron oportunidades a las pequeñas unidades económicas rurales. La implementación de una nueva política agropecuaria, sustentada en el proceso de apertura comercial y alineada a las tendencias de los mercados agroalimentarios globales, entró en contradicción con las formas tradicionales de operación de miles de empresas rurales, lo cual ocasionó su inviabilidad económica y eventual desaparición.

El errático desempeño de la ganadería serrana en Sonora en el último cuarto del siglo XX condujo a un panorama desalentador. Al iniciar el siglo XXI, la ganadería del DDR 145 estaba apenas sostenida por pequeñas unidades de conformación familiar, descapitalizadas y carentes de infraestructura productiva; su participación en la cadena productiva se limitaba a la producción de bovinos para la venta (Camou, 1998; Herrera y Salazar, 2015).

La demanda gremial para sortear los factores limitantes de su actividad productiva fundamental indujo a la Asociación Ganadera Local de Suaqui Grande (AGLSG) a finales de 2001 a buscar alternativas de solución. El ejercicio condujo a los pequeños criadores a identificar su problemática, que se resumen en:

a) La participación atomizada de los ganaderos en la producción y comercialización les impedía solventar los gastos de alimentación del núcleo familiar, al tiempo que requerían mantener a los animales en el agostadero durante el periodo de estiaje.

b) La necesidad de recursos conducía a establecer convenios desventajosos con acopiadores de ganado: se comprometían a la entrega del becerro una vez que estaba listo para el destete, a cambio de un pago por adelantado.

c) La permanencia en la actividad ganadera de los productores tradicionales estaba condicionada al mejoramiento de su posición en la cadena bovinos-leche-carne. 


\section{Apartado metodológico}

Se adoptó el diseño narrativo como técnica de investigación. Esta herramienta permite reconstruir de forma cronológica el fenómeno analizado a partir de sucesos relatados por los propios actores locales (Hernández, Fernández, Baptista, Menéndez y Mendoza, 2014). La unidad de análisis fue la Unión de Sociedades del Centro Los Soakis, conformada por 18 cooperativas aglutinadoras de 131 de los 316 productores que operaban en Suaqui Grande. Del total de cooperativas se eligió una muestra no probabilística discrecional conformada por 12 sociedades.

Para recabar la información se realizaron entrevistas semiestructuradas. De los entrevistados, $70 \%$ estuvo conformado por hombres y $30 \%$ por mujeres. Su edad promedio era de 55 años. Siete contaban con escolaridad básica (primaria y secundaria), tres con nivel medio y dos con estudios de licenciatura. Además, se complementó información aplicando el mismo tipo de instrumento a cinco actores clave: el jefe del DDR 145 Mazatán, el jefe del Centro de Atención para el Desarrollo Rural (CADER) con adscripción al distrito, el presidente de la Asociación Ganadera de Suaqui Grande (2000-2003), el director regional del Fideicomiso de Riesgo Compartido (FIRCO) y el técnico extensionista que brindó acompañamiento a los productores entre 2006 y 2015. Las secciones en las que se dividió la entrevista fueron:

a) Antecedentes de las unidades productivas tradicionales.

b) Surgimiento de cooperativas familiares.

c) Interacción institucional y aprendizaje.

d) Empoderamiento e innovaciones.

e) La conformación de la Unión de Sociedades del Centro Los Soakis, S. C. de R. L.

f) El Proyecto Estratégico de la Unión de Sociedades del Centro Los Soakis.

g) La transformación productiva.

Las entrevistas se llevaron a cabo en dos periodos: del 5 al 13 de mayo y del 8 al 12 de noviembre de 2017 y se efectuaron en las unidades productivas. Además, se obtuvo información mediante observación no participante en las áreas de producción, así como la redacción de un diario de campo y otro de los instrumentos señalados por Hernández, Fernández, Baptista, Menéndez y Mendoza (2014). Para identificar a los actores individuales, las fechas de su integración como organismos colectivos, el objeto de su constitución, la infraestructura de cada sociedad cooperativa, así como la dotación de recursos de acuerdo con sus necesidades específicas, se revisaron documentos y registros, como el acta constitutiva de la Unión de Sociedades del Centro Los Soakis, S. C. de R. L. de C. V. y el Proyecto estratégico Adquisición de Vientres Cargados e Infraestructura Ganadera de la Unión de Sociedades del Centro Los Soakis, S. C. de R. L. de C. V. 


\section{Innovación organizativa para el rescate de las unidades productivas tradicionales}

El proceso de discusión y toma de decisiones en el seno de la AGLSG entre 2001 y 2002 coincidió con el cambio de administración pública federal y la promesa del nuevo gobierno de impulsar la modernidad rural con fundamento en la Ley de Desarrollo Rural Sustentable (LDRS), que procuraba la incorporación de las zonas marginales (y de los sectores rurales más débiles en términos económicos) al desarrollo nacional (Diario Oficial de la Federación [DOF], 2001). En concordancia con este mandato de política pública, a finales de 2002 la Secretaría de Agricultura, Ganadería, Desarrollo Rural, Pesca y Alimentación (SAGARPA) estableció el Programa de Desarrollo de Capacidades en el Medio Rural (PRODESCA) (SAGARPA, 2013). Para incidir en la actividad de los grupos ganaderos del DDR 145, los operadores del Programa indujeron, a través de talleres participativos, diversos servicios de capacitación técnica, comercial y administrativa.

Para la AGLSG, esta coyuntura representó la oportunidad de acceso a la mezcla de recursos y apoyos dispuestos en las reglas de operación de las instituciones públicas de fomento rural y en la reglamentación de sus organismos gremiales. Esto alentó a su comité directivo ${ }^{2}$ a estructurar, en el seno de sus asambleas periódicas y reuniones de trabajo, un plan de rescate de las unidades productivas tradicionales, atendiendo a dos áreas detectadas como de alta prioridad para las empresas agremiadas: el abasto de agua y la innovación tecnológica para generación de valor adicional a los productos de la ganadería regional.

La primera acción de la AGLSG fue integrar los expedientes de sus asociados para incluirlos como sujetos de apoyo del PRODESCA. Las solicitudes se alinearon a incrementar la eficiencia en los métodos de extracción de agua en los ranchos, así como de su distribución en praderas, zonas forrajeras y áreas de manejo de ganado. En respuesta a sus gestiones, los asociados obtuvieron apoyos para desplegar dos grandes proyectos de impacto regional: la electrificación del riego por bombeo y un sistema de almacenamiento y conducción de agua.

La sustitución de motores de combustión interna (diésel o gasolina) por bombas eléctricas permitió a las unidades ganaderas reducir en $50 \%$ sus costos de producción de forraje e irrigación de praderas. El sistema de almacenamiento y conducción de agua implementado consistió en la creación de un reservorio de cinco pilas con capacidad de almacenaje superior a $300000 \mathrm{~m}^{3}$ de agua, ubicado en los puntos elevados de las comunidades y equipado con sistemas de bombeo electrificado. Estas obras aseguraron el abasto hídrico por gravedad a unidades ganaderas dispersas en un radio aproximado de $10 \mathrm{~km}$.

2 Comunicación personal en 2000 del ingeniero Jorge Luis Fimbres Castillo, presidente de la AGLSG y extensionista del Patronato del Centro de Investigaciones Pecuarias del Estado de Sonora (PATROCIPES) para pequeños ganaderos de Suaqui Grande a partir de 2003. 
El surgimiento de las cooperativas familiares

De conformidad con los acuerdos delineados en sus asambleas, la AGLSG procedería, en una segunda etapa, a gestionar una batería de proyectos de modernización tecnológica para la producción de leche y carne en las unidades económicas perfiladas como sujetos de apoyo en las reglas de operación de las instituciones de fomento rural. Al principio se preveía que la estrategia sería instrumentada con el soporte de los esquemas tradicionales de organización; sin embargo, entre los productores se perfiló un ambiente abierto de desconfianza frente a los líderes tradicionales que en el futuro apoyarían tales estructuras. En la opinión de los actores entrevistados, su resistencia reflejaba el descrédito de los grupos directivos tradicionales en su desempeño, frente a los embates tradicionales de la política económica y la cuestionable transparencia con que operaban los apoyos al campo en las décadas precedentes.

El clima de desacuerdo por mantener los modelos organizativos tradicionales condujo al comité directivo de la AGLSG a convocar a sus agremiados a una asamblea extraordinaria permanente, celebrada en junio de 2003, cuyo único punto fue el análisis y discusión de esquemas alternativos de organización coadyuvantes con la inducción de innovaciones en las unidades de producción.

En la discusión, los ganaderos concluyeron que la atención a su problemática requería la adopción de una estructura alineada con las prácticas que dieron soporte a sus estrategias de supervivencia, respetando usos y costumbres. Esta determinación se sustentó en dos reflexiones:

a) La conformación familiar explicaba la permanencia de las unidades productivas en un contexto económico hostil. Sin embargo, a ello también contribuía la arraigada interacción con otros actores de la comunidad que influían en el impulso de nuevos proyectos y acciones estratégicas de supervivencia basadas en la noción de que lo más importante no es el aporte económico sino el bienestar comunitario.

b) Las mismas estrategias de supervivencia que se basaban en la inercial operación de la empresa familiar, en gran medida explicada por su resistencia cultural a romper con usos y costumbres, también resultaban contradictorias en un contexto que retaba a los espacios locales a generar adaptaciones congruentes con las exigencias dinámicas de los mercados globales. Frente a esa realidad, el desarrollo de capacidades innovadoras se erigía como elemento esencial para la búsqueda de competitividad.

Las conclusiones dieron soporte a la adopción de un esquema de organización de empresas familiares aglutinadas en cooperativas, con base en su proximidad territorial, lazos de parentesco, afinidad en visiones y objetivos, entre otros factores de cohesión. En el segundo semestre de 2003 se constituyeron de forma legal las primeras cooperativas familiares (véase tabla 1), congregadas de manera informal en una estructura corporativa con dos propósitos específicos: el primero fue construir acuerdos estratégicos con el medio institucional para pasar de sólo criadores de becerros a las fases de acopio y comercialización 
de ganado en la cadena productiva bovino-leche-carne; el segundo, fortalecer las empresas rurales familiares con el impulso de alternativas de asociación y cooperación en redes que les aseguraran economías de escala y la creación y apropiación de una mayor proporción del valor agregado a los productos.

Tabla 1. Suaqui Grande: constitución de sociedades cooperativas familiares en 2003 y 2006

\begin{tabular}{|l|c|}
\hline \multicolumn{1}{|c|}{ Nombre de la cooperativa } & $\begin{array}{c}\text { Número de } \\
\text { Socios }\end{array}$ \\
\hline Ocho cooperativas constituidas en 2003 & 54 \\
\hline Sociedad Cooperativa Ganadería Integral El Tunal I & 8 \\
\hline Sociedad Cooperativa Bugamechin & 7 \\
\hline Sociedad Cooperativa Lucafrán & 6 \\
\hline Sociedad Cooperativa Zitotarais & 6 \\
\hline Sociedad Cooperativa La Pila de Gurrola & 6 \\
\hline Sociedad Cooperativa Los Vaqueros de San José de Milpilla & 7 \\
\hline Sociedad Cooperativa Represo de Cornelio & 7 \\
\hline Sociedad Cooperativa Carrizito de Gursa & 7 \\
\hline Once cooperativas constituidas en 2006 & 74 \\
\hline Sociedad Cooperativa El Arroyo Seco de la Cañada Embrujada & 8 \\
\hline Sociedad Cooperativa El Bajío de los Callejones & 7 \\
\hline Sociedad Cooperativa El Represo de Castillo & 6 \\
\hline Sociedad Cooperativa La Mesa de los Campa & 5 \\
\hline Sociedad Cooperativa Los Dikes del Pozo San José & 9 \\
\hline Sociedad Cooperativa Los Flores del Garabato & 5 \\
\hline Sociedad Cooperativa El Toquison de Navarro & 5 \\
\hline Sociedad Cooperativa El Chois del Pilar & 5 \\
\hline Sociedad Cooperativa Productores del Mátape & 12 \\
\hline Sociedad Cooperativa Las Tezotas de Tónichi & 6 \\
\hline Sociedad Cooperativa La Cañada de Catarina & 6 \\
\hline TOTAL & 128 \\
\hline
\end{tabular}

Fuente: acta constitutiva de la Unión de Sociedades del Centro Los Soakis, S. C. de R. L. de C. V.

La interacción institucional y la gestión de aprendizajes

Las cooperativas construyeron, bajo el liderazgo de la AGLSG, una red de vínculos interinstitucionales que facilitó su acceso a apoyos productivos y capacitación, así como a la implementación de innovaciones organizativas y tecnológicas de bajo costo, pero de alto impacto. La interacción con las instituciones públicas y con el sector de ciencia y tecnología permitió cerrar la brecha de 
competencias tecnológicas, organizativas y administrativas que limitaban su integración económica. En la percepción de los actores entrevistados destaca que las acciones que lideraron la estrategia de apropiación de la cadena de valor se basaron en nuevos conocimientos (capacitación), inductores de las innovaciones.

Los apoyos - ausentes en las décadas precedentes- empezaron a fluir a las unidades económicas familiares proveyéndolas de pequeñas obras de infraestructura de riego (pilas, tubería de conducción de agua, equipamiento de pozos), equipamiento de corrales de manejo (comederos, corrales, abrevaderos, cercados) e implementos para elaborar derivados lácteos (queso regional), entre las más frecuentes (véase tabla 2 ).

En el periodo 2003-2005, los apoyos productivos y los servicios de capacitación atendieron las necesidades de las cooperativas familiares con base en solicitudes específicas. Al mismo tiempo, la AGLSG gestionó servicios de capacitación en temáticas transversales, como innovación, sustentabilidad y formación de cultura empresarial. Los temas eran abordados en asambleas generales constituidas como espacios para la búsqueda de soluciones a problemas comunes. ${ }^{3}$

En 2004, los esfuerzos de gestión de la AGLSG ante el FIRA condujeron a la incorporación de las cooperativas en un programa de capacitación orientado a la innovación de prácticas de producción, suplementación, ordeña y manejo de ganado mediante la adopción de tecnología. En esa experiencia, los productores avanzaron en la apropiación del concepto de innovación como una combinación exitosa de tecnologías, conocimientos y esquemas mentales, cuya implementación requería nuevas formas de organización social. Los alcances de la innovación, además de la tecnología y la producción, incluyeron la organización en el sentido de actitudes, prácticas y nuevas formas de trabajar (Sonnino y Ruane, 2013).

En 2005, como parte de sus estrategias de integración de las cooperativas familiares y para fortalecer su interacción con instituciones gubernamentales y del sector de ciencia y tecnología, la AGLSG, con la ayuda del Fondo Nacional de Apoyo para las Empresas en Solidaridad (FONAES), propuso la adopción del modelo de Grupo Ganadero de Validación y Transferencia de Tecnología (GGAVATT). En este modelo de transferencia de tecnología a grupos de ganaderos organizados en torno a un módulo de validación, los recursos humanos especializados en extensionismo, desarrollo rural y agronegocios asumen el rol de agentes de cambio que acompañan a los productores, en coordinación con las instituciones oficiales del sector y los centros de investigación y tecnología. La metodología se orienta al fortalecimiento de la organización mediante la capacitación, validación, transferencia y adopción de tecnología para mejorar la productividad y competitividad de las unidades de producción (Ponce, Álvarez y Ceja, 2016).

3 Información obtenida en entrevista con el ingeniero Francisco Flores Lara, técnico extensionista asignado por el PATROCIPES a las comunidades de Los Soakis. 
Tabla 2. Innovaciones a partir de apoyos institucionales a las cooperativas familiares ganaderas de Suaqui Grande

\begin{tabular}{|c|c|c|c|c|}
\hline Institución & Programa & Proyecto Asociado & Innovación & Año \\
\hline $\begin{array}{c}\text { Financiera Nacional } \\
\text { de Desarrollo } \\
\text { Agropecuario, } \\
\text { Rural, Forestal y } \\
\text { Pesquero (FND) }\end{array}$ & $\begin{array}{l}\text { Formación, } \\
\text { capacitación y } \\
\text { consultoría para } \\
\text { productores e } \\
\text { intermediarios } \\
\text { financieros rurales }\end{array}$ & $\begin{array}{c}\text { Proyecto } \\
\text { Estratégico Servicios } \\
\text { de Consultoría }\end{array}$ & $\begin{array}{c}\text { Incorporacion de } \\
\text { prácticas basadas } \\
\text { en diagnósticos } \\
\text { participativos para la } \\
\text { adopcion estratégica de } \\
\text { servicios de consultoría }\end{array}$ & 2003 \\
\hline $\begin{array}{l}\text { Fideicomisos } \\
\text { Instituidos en } \\
\text { Relación con la } \\
\text { Agricultura (FIRA) }\end{array}$ & $\begin{array}{l}\text { Capacitación } \\
\text { especializada }\end{array}$ & $\begin{array}{l}\text { Capacitación } \\
\text { especializada para } \\
\text { innovar prácticas } \\
\text { de producción, } \\
\text { suplementación, } \\
\text { ordeña y manejo } \\
\text { de ganado } \\
\end{array}$ & $\begin{array}{c}\text { Adquisición de } \\
\text { competencias para } \\
\text { innovación de prácticas } \\
\text { de suplementación, } \\
\text { ordeña y manejo } \\
\text { de ganado }\end{array}$ & 2004 \\
\hline $\begin{array}{l}\text { Fideicomiso } \\
\text { de Riesgo } \\
\text { Compartido }\end{array}$ & $\begin{array}{c}\text { Nacional de } \\
\text { Microcuencas } \\
\text { Adquisición de } \\
\text { vientres cargados } \\
\text { e infraestructura } \\
\text { ganadera }\end{array}$ & $\begin{array}{c}\text { Plan Rector Producción } \\
\text { y Conservación } \\
\text { territorial sustentable } \\
\text { (proyectos específicos) }\end{array}$ & $\begin{array}{l}\text { Mejoramiento genético } \\
\text { vía adquisición de } \\
\text { vientres preñados de } \\
\text { ganado certificado } \\
\text { Incorporación de } \\
\text { infraestructura para } \\
\text { innovar suplementación, } \\
\text { ordeña y manejo de } \\
\text { ganado certificado }\end{array}$ & 2005 \\
\hline SAGARPA & $\begin{array}{c}\text { Modelo de } \\
\text { Grupo Ganadero } \\
\text { de Validación y } \\
\text { Transferencia de } \\
\text { Tecnología }\end{array}$ & $\begin{array}{l}\text { Módulos de validación } \\
\text { para adopción de } \\
\text { tecnología generada } \\
\text { en campos } \\
\text { experimentales }\end{array}$ & $\begin{array}{c}\text { Adopción de nuevas } \\
\text { tecnologías generadas } \\
\text { en campos } \\
\text { experimentales propios }\end{array}$ & 2005 \\
\hline $\begin{array}{c}\text { Comisión Nacional } \\
\text { de Zonas Áridas } \\
\text { (CONAZA) } \\
\text { FONAES }^{1}\end{array}$ & $\begin{array}{c}\text { Repoblamiento } \\
\text { del hato } \\
\text { Ganadero }\end{array}$ & $\begin{array}{c}\text {-Molinos forrajeros } \\
\text {-Equipamiento de pozo } \\
\text { comederos, corrales, } \\
\text { salas de ordeña, pila, } \\
\text { tuberías } \\
\text {-Capacitación } \\
\text { especializada }\end{array}$ & $\begin{array}{c}\text { Renovación de equipo: } \\
\text {-Molinos forrajeros } \\
\text {-Equipamiento: pozos, } \\
\text { comederos y corrales, } \\
\text { Adopción de salas de } \\
\text { ordeña mecanizadas } \\
\text { Capacitación en su uso y } \\
\text { mantenimiento }\end{array}$ & $\begin{array}{l}2004 \\
2005 \\
2005\end{array}$ \\
\hline $\begin{array}{c}\text { Patronato del } \\
\text { Centro de } \\
\text { Investigaciones } \\
\text { Pecuarias del } \\
\text { Estado de Sonora, } \\
\text { A C (PATROCIES) }\end{array}$ & $\begin{array}{l}\text { Desarrollo } \\
\text { de Proyectos } \\
\text { Agropecuarios } \\
\text { Integrales } \\
\text { (DEPAl) }\end{array}$ & $\begin{array}{c}\text {-Extensionismo } \\
\text {-Capacitación para } \\
\text { elevar la eficiencia } \\
\text { productiva con } \\
\text { innovación tecnológica } \\
\text { a bajo costo e impactos } \\
\text { a corto plazo }\end{array}$ & $\begin{array}{c}\text { Adopción de prácticas } \\
\text { tecnológicas innovadoras } \\
\text { de bajo costo para } \\
\text { incrementar la eficiencia } \\
\text { del sistema productivo }\end{array}$ & 2005 \\
\hline $\begin{array}{c}\text { Instituto Nacional } \\
\text { de Investigaciones } \\
\text { Forestales, } \\
\text { Agrícolas y } \\
\text { Pecuarias }\end{array}$ & $\begin{array}{l}\text { Capacitación y } \\
\text { extensionismo }\end{array}$ & $\begin{array}{c}\text {-Inseminación artificial } \\
\text {-Destete precoz }\end{array}$ & $\begin{array}{l}\text { Adopción de prácticas } \\
\text { especializadas de } \\
\text { inseminación artificial } \\
\text { y destete precoz }\end{array}$ & $\begin{array}{l}2003 \\
2005\end{array}$ \\
\hline
\end{tabular}

Fuente: elaboración propia a partir de entrevistas semiestructuradas.

1 Fondo Nacional de Apoyo para las Empresas en Solidaridad. 
Empoderamiento e innovaciones

La estrategia de interacción institucional se realineó en asambleas periódicas de capacitación y organización de las cooperativas y grupos de productores independientes congregados en GGAVATT. Las reuniones eran conducidas con base en disposiciones reglamentarias incuestionables de respeto y tolerancia, junto a la representación de las cooperativas familiares. Actores del sector público o académico asistían por invitación para atender a las necesidades específicas de información y capacitación a todos los asistentes.

En esos años, la asistencia técnica fue reforzada por el PATROCIPES al asignar los servicios de un técnico extensionista, responsable de apuntalar las acciones de capacitación, validación, transferencia y adopción de tecnologías de conformidad con los propósitos del GGAVATT. Este apuntalamiento fortaleció las gestiones de la AGLSG y el liderazgo de las cooperativas en su acceso a recursos del FONAES para la instalación de ocho salas de ordeña mecanizada, conforme lo estipulaba su estrategia de adopción de capacidades tecnológicas para elevar la eficiencia productiva y la generación de valor.

Al ser analizados en las reuniones del GGAVATT los factores que condujeron a la mecanización de la ordeña en las cooperativas, no pasó inadvertido que éstas captaban los apoyos públicos y el acompañamiento de la comunidad científica. Ello ocurría en un contexto donde, además del territorio y la condición socioeconómica, las cooperativas compartían con el resto de los asociados del GGAVATT las mismas oportunidades de gestión de capacitación y acceso a apoyos públicos. La explicación atribuida a este fenómeno se resumía en tres factores:

a) El grado de cohesión que las estructuras familiares presentaban en alianza con la AGLSG en sus gestiones frente a las instituciones de fomento rural.

b) Su interacción ofrecía ventajas en el momento de establecer liderazgos en los temas de análisis y los procesos de toma de decisiones, tanto a escala de su participación en el GGAVATT en lo particular, como en la AGLSG en lo general.

c) El flujo de información e intercambio de experiencias en su práctica de apropiación del conocimiento (capacitación especializada) e implementación en la solución de problemas comunes.

Alentados por las conclusiones de su análisis, se generalizó entre los asociados del GGAVATT el interés en adoptar el esquema de las cooperativas familiares y las prácticas de acuerdos y alianzas que las organizaba como conglomerado de empresas familiares. Ello condujo a que en el transcurso de 2006 se constituyeran 11 cooperativas adicionales a las ya existentes.

\section{La conformación de la Unión de Sociedades del Centro y su estrategia para enfrentar la crisis alimentaria global}

El aprovechamiento de las redes interinstitucionales permitió a las cooperativas establecer fortalezas internas para dar impulso a iniciativas de integración 
económica de la actividad ganadera. La pertinencia de esos propósitos se confirmó ante la manifestación de la crisis alimentaria entre 2007 y 2008, coincidente con el repunte de las crisis financiera y energética global, todo ello en un entorno de deterioro ambiental incidente en la producción, distribución y consumo de alimentos (Paz, 2015).

En México, la crisis incidió sobre un sector agropecuario frágil y polarizado. El repunte de los precios de los granos no generó mayores beneficios a los agricultores e impactó de forma negativa en la economía de los ganaderos, quienes no habían logrado sobreponerse a los efectos del modelo económico neoliberal implementado en la década de 1980 (Rubio, 2013). En algunos estudios consta la disminución en 2010 en $22 \%$ de las unidades de producción ganaderas con respecto al censo de 1991 (Rubio, 2013).

Frente a las manifestaciones de la crisis alimentaria, financiera y energética sobre los sistemas agroalimentarios locales, las cooperativas antepusieron acciones corporativas de contención de impactos. En este punto, con el objetivo de formar capacitadores y consultores rurales para incidir en el desarrollo de competencias de los productores, apoyados por el Colegio de Postgraduados (COLPOS), la Financiera Nacional de Desarrollo (FND) y el Centro de Investigación en Alimentación y Desarrollo (CIAD), se inició un programa de Maestría Tecnológica en Prestación de Servicios Profesionales enfocado en las actividades rurales. Con estas alianzas, se procedió a diseñar un proyecto estratégico que permitiera integrarse de manera competitiva a los eslabones de la cadena productiva bovinos-leche-carne. Como parte de esta estrategia, en 2009 se constituyeron de forma legal en la Unión de Sociedades del Centro Los Soakis, S. C. de R. L., con una membresía de 19 sociedades cooperativas que aglutinaba a 130 ganaderos y sus familias.

En su objeto social, Los Soakis imprimieron una perspectiva de desarrollo sustentada en la acción planificada de diseño, incubación y fortalecimiento de empresas estructuradas bajo una dirección corporativa con administraciones independientes y autónomas, para generar vínculos de valor en los eslabones de la cadena productiva: abasto, acopio, almacén, transporte, comercialización, servicios financieros. Y entre los objetivos específicos destacaban:

1. Impulsar la consolidación de las unidades de producción para su incorporación al eslabón primario a través de la creación de infraestructura, equipamiento y adquisición de ganado de doble propósito.

2. Habilitar las unidades productivas en prácticas de acopio de leche y fabricación de sus derivados para promover el ingreso a la cadena de valor como proveedoras directas del mercado.

3. Asegurar la competitividad de las empresas integrando los procesos de acopio, recría, engorda, sacrificio y ventas directas de ganado y carne.

4. Generar economías de escala en las unidades productivas y capitalizar en favor de la organización la colocación de servicios diversos (maquilado, apoyo técnico) y provisión de insumos al mercado regional. 
La iniciativa planificada se tradujo en la gestión del proyecto estratégico Unión de Sociedades del Centro Los Soakis, S. C de R. L. de C. V. ante las instituciones de Fomento Rural lideradas por SAGARPA. En él se contempló la inversión en activos productivos para la constitución de cinco unidades de negocios que dieran fundamento a la integración de las cooperativas familiares a los eslabones de la cadena bovinos-leche-carne. Estas unidades de negocios fueron: un centro de acopio y procesamiento de queso, una distribuidora de insumos, un centro de recría, un centro de acopio de carne y una unidad dispersora de crédito.

\section{El Proyecto Estratégico de Integración Económica}

En 2010, dando crédito a la importancia estratégica que para la ganadería regional representaban las innovaciones impulsadas por Los Soakis, la SAGARPA estableció, en la Sociedad Cooperativa Lucafrán, un rancho demostrativo de 500 hectáreas, dividido en 40 potreros, para la difusión de prácticas sustentables en el manejo de agostadero, ganado de doble propósito y producción de queso. La actividad demostrativa atrajo la atención de dos tipos de visitantes: la comunidad científica y el sector productivo. Los miembros de la primera, además de corroborar los resultados de la implementación de innovaciones, enfocaban su interés en las especies vegetales amenazadas o en peligro de extinción, beneficiadas por el manejo responsable de los recursos implementados por Los Soakis; el segundo grupo se interesó en recibir capacitación en prácticas productivas y organizativas (véase tabla 3 ).

El desempeño de las comunidades Soakis corroborado en las instalaciones de la cooperativa Lucafrán disipaba la preocupación de las instituciones de fomento rural por identificar un modelo capaz de atenuar los efectos negativos de la crisis alimentaria, financiera y energética sobre los sistemas agroalimentarios locales. La SAGARPA designó un grupo de expertos para determinar el potencial de difundir las innovaciones de Los Soakis. ${ }^{4}$ Un reporte de la Subsecretaría de Desarrollo Rural recomendó incrementar la escala de las operaciones a través de un proyecto estratégico de integración económica denominado Adquisición de Vientres Cargados e Infraestructura Ganadera de la Unión de Sociedades del Centro Los Soakis, S. C. de R. L. de C. V. (FIRCO, 2011). Esta recomendación de la SAGARPA fue respaldada por el FIRCO y por el Programa Nacional de Microcuencas con la aportación de 10.28 millones de pesos para la adquisición de 340 vaquillas preñadas de las razas pardo suiza europea, pardo suiza americana y jersey, 17 sementales y la construcción de obras de infraestructura agropecuaria, apalancando el proyecto estratégico desagregado en los diferentes rubros descritos en la tabla 4.

4 Comunicación personal con el ingeniero Adalberto Gutiérrez Morales, coordinador del Programa de Microcuencas asignado por FIRCO para la instrumentación del proyecto Adquisición de Vientres Cargados e Infraestructura Ganadera de la Unión de Sociedades del Centro los Soaquis, S. C. de R. L. de C.V. 
Tabla 3. Grupos de visitantes permanentes del rancho demostrativo de la Sociedad Cooperativa Lucafrán

Miembros de la comunidad científica

Internacionales:

- Grupos académicos procedentes de universidades de California y Arizona, EE. UU.

- Universidad de Arizona y Universidad Estatal de Arizona.

- Universidad de California San Diego (La Jolla).

- Universidad Politécnica del Estado de California (Cal Poly).

Nacionales:

- Universidad Nacional Autónoma de México.

- Universidad Autónoma de Chapingo.

- Colegio de Postgraduados (COLPOS).

- Universidad Autónoma de Baja California.

Regionales:

- Centro de Investigación en Alimentación y Desarrollo, A. C.

- El Colegio de Sonora.

- Universidad de Sonora.

- Universidad Estatal de Sonora.

Observadores del sector productivo

Productores pecuarios de doble propósito de Sonora:

- Actores de los Distritos de Desarrollo Rural: 139-Caborca, 146-Sahuaripa, 147-Guaymas-Empalme, 139-Magadalena, son capacitados en prácticas de recuperación del agostadero basadas en pastoreo controlado.

- Ganaderos pecuarios del DDR 142-Ures observan y adaptan el esquema organizativo de cooperativas familiares para fortalecer su propia estructura.

- Grupos de mujeres del DDR 145 se incorporan de manera activa a las jornadas de capacitación para la producción bajo ambiente controlado en sus municipios de origen.

Fuente: elaboración propia a partir de entrevistas semiestructuradas.

Tabla 4. Proyecto estratégico Unión de Sociedades del Centro Los Soakis, S. C. de R. L. de C. V. Rubros de inversión por fuente de financiamiento

\begin{tabular}{|c|c|c|c|c|c|c|c|c|c|}
\hline Concepto & Descripción & Cantidad & \multicolumn{6}{|c|}{ Aportación (en miles de pesos) } \\
\cline { 4 - 9 } & & Federal & $\%$ & Estatal & $\%$ & Usuarios* & $\%$ & Total \\
\hline $\begin{array}{c}\text { Adquisición } \\
\text { de ganado }\end{array}$ & $\begin{array}{c}\text { Vaquillas } \\
\text { Toros }\end{array}$ & 34017 & 4293.54 & 66 & 1178.10 & 18 & 1073.36 & 16 & 6545.0 \\
\hline
\end{tabular}




\begin{tabular}{|c|c|c|c|c|c|c|c|c|c|}
\hline \multirow{8}{*}{ Infraestructura } & Comederos & $295.5 \mathrm{~m}$ & \multirow[t]{8}{*}{889.17} & \multirow[t]{8}{*}{42} & \multirow[t]{8}{*}{545.47} & \multirow[t]{8}{*}{25} & \multirow[t]{8}{*}{706.62} & \multirow[t]{8}{*}{33} & \multirow[t]{8}{*}{2141.26} \\
\hline & \multicolumn{2}{|l|}{$\begin{array}{c}\text { Corral } \\
\text { de manejo }\end{array}$} & & & & & & & \\
\hline & $\begin{array}{l}\text { Sombra } \\
\text { de corral }\end{array}$ & 8 & & & & & & & \\
\hline & Almacén & 3 & & & & & & & \\
\hline & $\begin{array}{l}\text { Sala de } \\
\text { ordeña }\end{array}$ & 10 & & & & & & & \\
\hline & Conducción & $3.6 \mathrm{~km}$ & & & & & & & \\
\hline & Pilas & 5 & & & & & & & \\
\hline & Pozo & 1 & & & & & & & \\
\hline & Ordeña & 10 & 600.25 & 50 & 204.09 & 17 & 396.17 & 33 & 1200.50 \\
\hline & $\begin{array}{c}\text { Molino } \\
\text { forrajero }\end{array}$ & 4 & & & & & & & \\
\hline Equipamiento & Mezcladora & 1 & & & & & & & \\
\hline & Pozo & 3 & & & & & & & \\
\hline & \begin{tabular}{|c|c|}
$\begin{array}{c}\text { Calentador } \\
\text { solar }\end{array}$ \\
\end{tabular} & 1 & & & & & & & \\
\hline $\begin{array}{c}\text { Capital de } \\
\text { trabajo }\end{array}$ & $\begin{array}{l}\text { Cultivo } \\
\text { de alfalfa }\end{array}$ & 14 ha & & & & & 114.80 & 100 & 114.80 \\
\hline Seguro & & & & & & & 279.27 & 100 & 279.27 \\
\hline Total & & & 5782.97 & & 1927.66 & & 2570.21 & & 10280.83 \\
\hline
\end{tabular}

*Aportación financiera del Fondo para las Actividades Productivas del Estado de Sonora (FAPES). Fuente: FIRCO (2011).

\section{Algunos resultados del proceso de transformación productiva}

En el periodo 2010-2015, la ganadería sonorense experimentó una sensible reducción de su hato estimada en 14\% (véase figura 2). Este quebranto se atribuyó a los efectos de la crisis financiera, energética y ambiental que a escala global expuso la fragilidad de los sistemas agroalimentarios locales a partir de 2007. A escala regional, las manifestaciones de este fenómeno se profundizaron estimuladas por la recurrente sequía, la contracción del financiamiento público-privado y el encarecimiento de los insumos (Andablo, Hernández y Catalán, 2015; Herrera y Salazar, 2015; Paz, 2015). El impacto de la profundización de la crisis económica sobre el precio internacional de los alimentos impulsó la cotización regional del ganado y de la carne e incidió en el aumento del valor nominal del hato sonorense en $36 \%$.

Los informes de las subastas celebradas en la Unión Ganadera Regional de Sonora (UGRS) de 2017 confirman que, entre enero de 2010 y diciembre de 2015, el precio del ganado en pie presentó aumentos sin precedentes de $29 \%$ anual en promedio, para un total acumulado de 179\%. Los informes también 
documentan la notable diversificación de la demanda de ganado para carne en todas sus calidades y no sólo para el de exportación, así como la combinación de este fenómeno con el deterioro económico que para los pequeños criadores representó la mortandad de reses propiciada por la prolongada sequía y la consecuente escasez de pastos.

En el DDR 145, los efectos de la crisis redujeron el stock bovino en $31 \%$. No obstante, la trayectoria ascendente de precios incrementó el valor de su inventario en 33\% (véase figura 2). Villa Pesqueira y San Javier registraron pérdidas absolutas en el tamaño y valor del hato. El resto de los municipios, aunque favorecidos por el incremento de los precios, experimentaron la disminución de sus reservas. Suaqui Grande observó la disminución de $41 \%$ de su ganado, la mayor en la región, mientras que reportó un incremento en el valor de producción de $15 \%$.

Figura 2. Cambios en el volumen y valor de la producción de ganado en pie durante 2010-2016 en Sonora y en los municipios del DDR 145

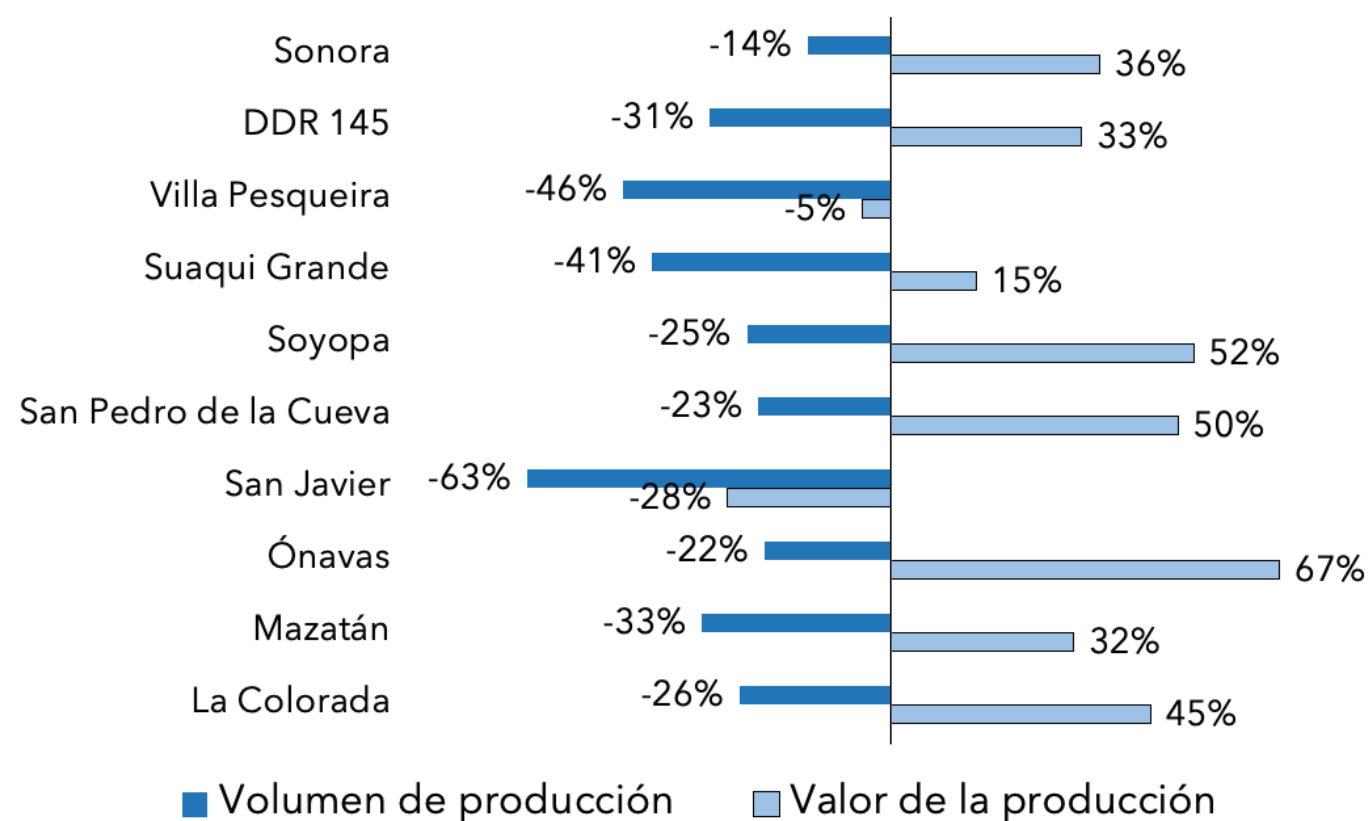

Fuente: elaboración propia con base en Sistema de Información Agroalimentaria y Pesquera (SIAP) (2019).

Sin embargo, mientras que a escala del DDR-145 (y del estado de Sonora en su conjunto) las causas de la disminución del hato fueron atribuidas a la sequía extrema y a la escasez de pastos, en las comunidades de Suaqui Grande fueron producto de las estrategias de depuración genética previstas por la AGLSG. Esos esfuerzos estaban alineados con el liderazgo de las comunidades Soakis deter- 
minado a lograr la conformación de un hato menos vulnerable a los efectos del cambio climático, de conformidad con lo previsto en su proyecto estratégico de integración económica de la ganadería de doble propósito.

Las secuelas de la crisis multifactorial fueron aún más profundas en la actividad lechera sonorense que en la cría de ganado para carne: durante el periodo 2010-2015, los sistemas productivos lecheros del estado reportaron un quebranto de $10 \%$ en el volumen y de $21 \%$ en el valor de la producción (véase figura 3 ). Las percepciones de los actores de la ganadería en el DDR 145 indican que las enormes pérdidas reportadas, aunque relacionadas con la disminución de las reservas de agua y pastos, se debieron a la venta de hembras, lo que se tradujo en la pérdida de valiosos vientres con los que los criadores solían dar soporte a la reproducción del hato, producción de leche y queso, sus principales y más regulares fuentes de ingresos.

La actividad lechera del DDR 145 presenta un crecimiento promedio de 8 y $15 \%$ en el volumen y valor de la producción, respectivamente, para el periodo 2010-2015. Sin embargo, la mayoría de sus municipios reportaron enormes pérdidas y parálisis en sus sistemas productivos, como es el caso de La Colorada, Soyopa, Villa Pesqueira, San Pedro de la Cueva y San Javier, que se vieron forzados a suspender la producción de queso ante la escasez de leche. Las aportaciones al saldo positivo expresadas en los indicadores a escala distrital derivaron de modo marginal del sistema productivo lechero del municipio de Mazatán, con incrementos de 10\% del volumen y $19 \%$ del valor de la producción, pero se debió al dinamismo de los sistemas productivos de Los Soakis, que en ese periodo mostraron un incremento de $86 \%$ y $99 \%$ en el volumen y valor de la producción de leche, respectivamente (véase figura 3 ).

En el DDR 145, Los Soakis constituyen el caso más integrado de la cadena de valor bovinos-carne-leche. Entre 2010 y 2015, sus actores pasaron de los esquemas extensivos tradicionales, que les aseguraban 55 pariciones como máximo de cada 100 vientres, comprometidos en venta al alcanzar alrededor de $75 \mathrm{~kg}$, a otro de tipo intensivo operado en unidades tecnificadas, donde por cada 100 vientres la parición promedio del hato ascendía a 75 crías que reportaban entre 180 y $200 \mathrm{~kg}$ de peso en el momento de su venta. En 2015, en esta cadena productiva se identifica la participación de 316 ganaderos que operaban 110 establecimientos; 44 productores en igual número de unidades concentraban un hato de 818 reses que generaba 997400 litros anuales de leche. Así mismo, en las labores de engorda concurrían 272 productores en 66 establecimientos económicos aportantes de 125.2 toneladas de carne en canal.

El escenario económico que han construido las comunidades Soakis es contrastante con los efectos que la crisis económica imprimió a la actividad ganadera sonorense en el período 2010-2015. El predominio de sus actores en los eslabones de la cadena de valor bovinos-leche-carne y el posicionamiento de sus productos en el mercado se ha erigido de conformidad con la inducción de innovaciones en su organización, equipamiento e infraestructura, entre otros rubros trascendentales, pero en especial en sus visiones de desarrollo. 
Figura 3. Cambios en el volumen y valor de la producción de leche entre 2010 y 2016 en Sonora y en los municipios del DDR 145

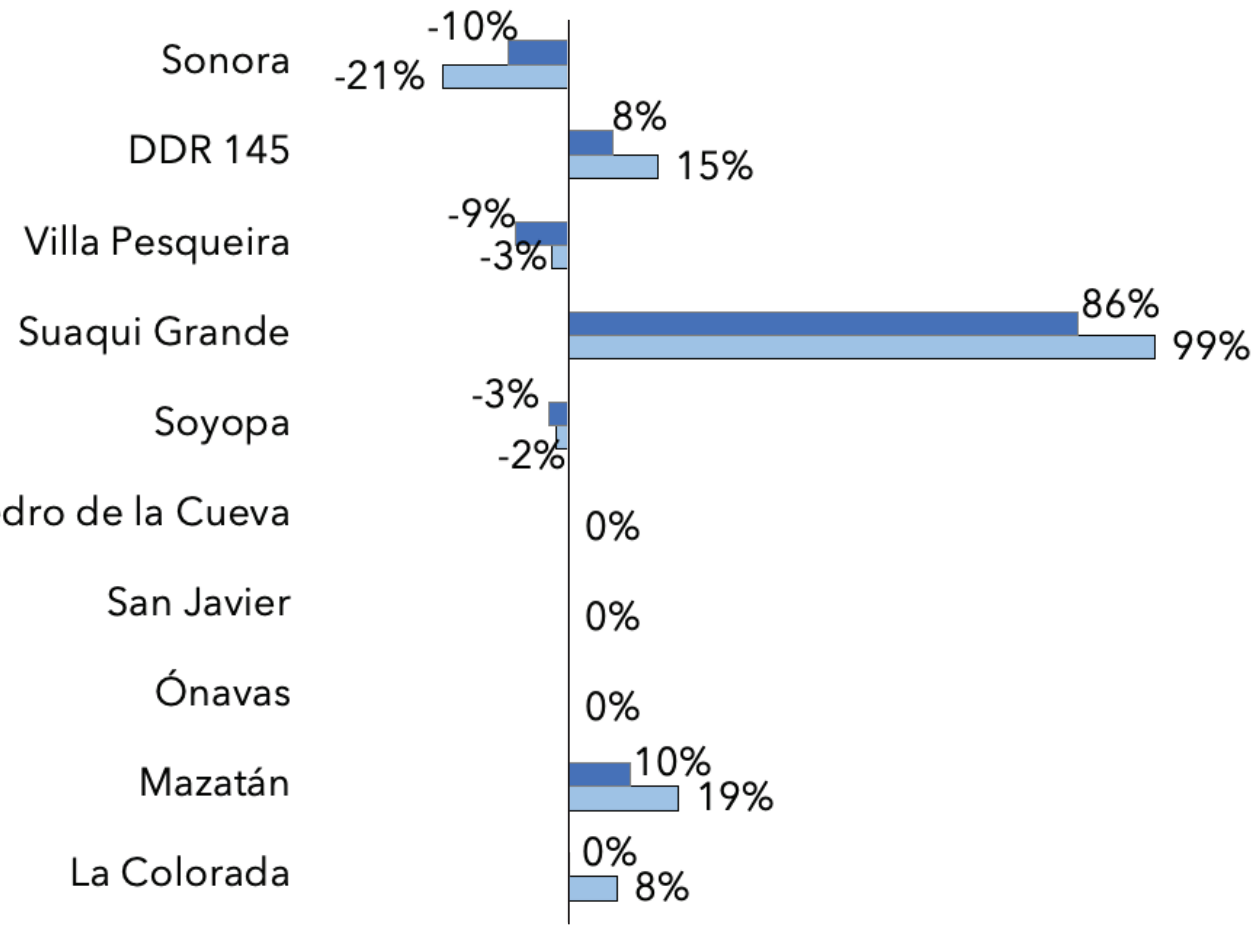

Volumen de producción $\quad \square$ Valor de la producción

Fuente: elaboración propia con base en SIAP (2019).

\section{Conclusiones}

En la experiencia de las comunidades Soakis, el desarrollo de su organización fue inducido con base en la reflexión de los problemas enfrentados en los procesos de trabajo donde lo trascendente, además de la manera en que estos sujetos se apropiaron de los aprendizajes individuales, fue la forma en que éstos penetraron en el sistema organizativo hasta lograr definir su funcionamiento (Kooiman y Jentoft, 2009). La visión compartida sobre la problemática de su sistema productivo permitió una respuesta coordinada de los actores y generó un proceso de gobernanza capaz de incidir en la difusión de innovaciones y el conocimiento sustentado en la base institucional de la cultura local. Por otro lado, hay que destacar que los asociados de las comunidades Soakis reportan 55 años como edad promedio, lo cual permite descartar una relación inversa entre adopción de innovaciones y edad, tal y como fue identificada por Cárde- 
nas-Bejarano, Gallardo-López, Núñez-Espinoza, Asiaín-Hoyos, Rodríguez-Chessani y Velásquez-Beltrán (2016) en los sistemas de doble propósito en Veracruz y Tampico, incorporados al modelo GGAVATT.

Las empresas familiares constituyen para Los Soakis la base de una estructura organizativa superior que les permitió acceder a los apoyos y el financiamiento necesarios para impulsar su proyecto de integración económica. Esto es consistente con los reportes de Rangel et al. (2017), quienes advierten sobre la condicionalidad que para la sostenibilidad de los sistemas de doble propósito del trópico mexicano representa su vinculación al sector de ciencia y tecnología, así como el soporte técnico público-privado con presencia en todas las fases del proyecto y tecnologías a implementar, incluido su acceso a financiamiento.

La constitución de sociedades cooperativas familiares ha fructificado en la participación de actores rurales tradicionales como empresarios al incorporar visiones que aportan innovaciones cimentadas por vínculos de confianza entre actores (públicos y privados) que conforman redes de gobernanza, identificadas desde la óptica de Estévez (2013) y Sørensen y Torfing (2016) como escenarios potenciales de innovación colaborativa.

El alcance de las transformaciones inducidas por Los Soakis corrobora que el aprendizaje y las destrezas obtenidas, su evolución y la manera de utilizarlos en el logro de sus objetivos dependen del tipo de conocimiento adquirido y de la forma en que lo han procesado, lo que North (2010) describe como un proceso incremental de sus capacidades y eficiencia adaptativa para la solución de problemas y la inducción de innovaciones. La evidencia de cambios incrementales en las capacidades de Los Soakis, capitalizadas en la solución de problemas a través de la inducción de innovaciones, es congruente con lo expresado por Vázquez-Barquero (2018), en el sentido de que las formas de organización crean un entorno institucional que condiciona los resultados económicos al definir las reglas sociopolíticas que regulan el comportamiento de los actores económicos y sus organizaciones.

En el desarrollo del sistema productivo de Los Soakis se advierte una fuerte presencia institucional que, sumada a la disposición de los actores productivos para coordinarse de manera constructiva en torno a proyectos de interés común, permite la solución de sus problemas. Ello promueve la conformación de un sistema de organización en redes, el cual Fukuyama (2016) reconoce como la guía para la interacción y la implementación de acciones desde lo local. El buen funcionamiento y la interacción de la red de actores e instituciones reducen los costos de información e incertidumbre y facilitan la toma de decisiones. $\mathrm{El}$ aprendizaje contribuye a la obtención de mejores resultados.

La asociación positiva entre la adopción de tecnología, el incremento de la red y la interacción social identificada por Cárdenas-Bejarano et al. (2016) coincide con lo observado en el proceso de adopción tecnológica en las comunidades Soakis. Así mismo, se detectó una marcada relación positiva entre educación, adquisición de competencias y adopción de innovaciones.

En concordancia con North (2010), el análisis confirma que al reducir la incertidumbre y proporcionar una estructura estable a la interacción humana, las instituciones determinan las oportunidades. Las organizaciones se crean para 
aprovecharlas con el deliberado propósito de lograr determinados objetivos, y en ese proceso las organizaciones se constituyen en fuente de cambio institucional. En el caso estudiado, la vinculación y el acompañamiento de las instituciones fueron fundamentales para el proceso de desarrollo territorial, con la presencia del PATROCIPES, CIAD y COLPOS para la capacitación, adaptación y difusión de tecnologías, lo cual propició la construcción de un capital humano capaz de activar los recursos territoriales y de avanzar en la consolidación de los sistemas productivos pecuarios.

\section{Referencias}

Alburquerque, F. (2017). Productividad, competitividad y políticas de desarrollo productivo territorial. Recuperado de https://www.delalburquerque.es/ wp-content/uploads/2017/12/Productividad-competitividad-y-DET.pdf

Andablo, A., Hernández, M., y Catalán, C. (2015). Gobernanza e integración de familias rurales a cadenas pecuarias: el caso del ejido Cobachi, Sonora. Economía: Teoría y Práctica, (42), 105-135. Recuperado de http://www.scielo. org.mx/pdf/etp/n42/n42a5.pdf

Boucher, F., y Reyes-González, J. (2016). El Enfoque SIAL como catalizador de la acción colectiva: casos territoriales en América Latina. Estudios Sociales 25(47), 13-37. Recuperado de https://www.ciad.mx/estudiosociales/index. $\mathrm{php} / \mathrm{es} /$ article/view/307/194

Camou, E. (1998). De rancheros, poquiteros, orejanos y criollos. Los productores ganaderos de Sonora y el mercado internacional. México: El Colegio de Michoacán y Centro de Investigación en Alimentación y Desarrollo.

Cárdenas-Bejarano, E., Gallardo-López, F., Núñez-Espinoza, J. F., Asiaín-Hoyos, A., Rodríguez-Chessani, M. A., y Velásquez-Beltrán, L. G. (2016). Redes de innovación en los grupos ganaderos de validación y transferencia de tecnología en México. Agricultura, Sociedad y Desarrollo, 13(2), 237-255.

Consejo Estatal de Población Sonora (COESPO). (2019). Indicadores sociodemográficos. Recuperado de http://www.coespo.sonora.gob.mx/indicadores. html

Diario Oficial de la Federación (DOF). (1988). Ley de Distritos de Desarrollo Rural. Recuperado de http://dof.gob.mx/nota_detalle.php?codigo=4718563\&fecha $=28 / 01 / 1988$

Diario Oficial de la Federación (DOF). (2001). Ley de Desarrollo Rural Sustentable (última modificación 12 de abril de 2019). Recuperado de http://www. diputados.gob.mx/LeyesBiblio/pdf/235_120419.pdf

Estévez, J. (2013). La influencia del neoinstitucionalismo en el discurso de la gobernanza. Mientras Tanto, (120), 83-109. Recuperado de https://www. jstor.org/stable/43491784?seq=1\#metadata_info_tab_contents

Farinós, J. (2005). Nuevas formas de gobernanza para el desarrollo sostenible del espacio relacional. Ería, (67), 219-235. Recuperado de https://www. unioviedo.es/reunido/index.php/RCG/article/view/1514/1430 
Fideicomiso de Riesgo Compartido (FIRCO). (2011). Convenio de Colaboración SAGARPA-Municipio de Suaqui Grande Sonora. Proyecto Institucional de concurrencia territorial-Microcuencas Adquisición de vientres cargados e infraestructura ganadera de la Unión de Sociedades del Centro Los Soakis, S. C. de R. L. de C. V. Hermosillo, México.

Fukuyama, F. (2016). Governance: What do we know, and how do we know it? Annual Review of Political Science, (19), 89-105. doi: https://doi. org/10.1146/annurev-polisci-042214-044240

Hernández, R., Fernández, C., Baptista, M., Menéndez, S., y Mendoza, C. (2014). Metodología de la investigación. México: McGraw Hill.

Herrera, R., y Salazar, V. (2015). Aprendiendo a exportar ganado: asesoría y financiamiento con los pequeños criadores de la sierra de Álamos. México: Colegio de Postgraduados y Financiera Nacional de Desarrollo.

Instituto Nacional de Estadística y Geografía (INEGI). (2015). Anuario estadístico y geográfico de Sonora 2015. Recuperado de https://www.inegi.org.mx/ app/biblioteca/ficha.html?upc=702825076313

Kooiman, J., y Jentoft, S. (2009). Meta-governance: values, norms and principles, and the making of hard choices. Public Administration, 87(4), 818-836. doi: https: / /doi.org/10.1111/j.1467-9299.2009.01780.x

North, D. (1993). Instituciones, cambio institucional y desempeño económico. México: Fondo de Cultura Económica.

North, D. (2010). Los andamios que el ser humano erige. En R. Vergara (comp.), Organización e instituciones (pp. 142-162). México: Siglo XXI Editores.

Organización para la Cooperación y el Desarrollo Económicos (OCDE). (2005). Manual de Oslo: guía para la recogida e interpretación de datos sobre innovación. Recuperado de http://www.itq.edu.mx/convocatorias/manualdeoslo.pdf

Paz, F. (octubre, 2015). Seguridad alimentaria y organizaciones rurales. Trabajo presentado en XX Congreso Internacional de Contaduría, Administración e Informática. Facultad de Contaduría y Administración, UNAM, México D. F. Recuperado de http://congreso.investiga.fca.unam.mx/docs/xx/ docs/10.25.pdf.

Ponce, F. Álvarez, D., y Ceja, L. (2016). Modelo GGAVATT y redes de innovación en la cuenca lechera Ciénega de Chapala, Michoacán. Revista Mexicana de Ciencias Agrícolas, 7(3), 545-558. doi: https://doi.org/10.29312/remexca. v7i3.308

Rangel, J., Espinosa, J. A., de Pablos-Heredero, C., Barba, C., Vélez, A., Rivas, J., y García, A. (2017). Adopción de innovaciones y prácticas organizativas de manejo, alimentación y reproducción en pequeñas unidades de producción de vacunos de doble propósito en México. Revista Científica, 27(1), 44-55.

Rubio, B. (2013). Impacto de la crisis alimentaria en la ganadería productora de carne. En Autor (coord.), La crisis alimentaria mundial: impacto sobre el campo mexicano (pp. 225-254l). México: Porrúa.

Secretaría de Agricultura, Ganadería, Desarrollo Rural, Pesca y Alimentación (SAGARPA). (2013). Convocatoria 2013 Formación en línea a PSP en los servicios básicos. Recuperado de https://www.gob.mx/cms/uploads/attachment/file/157703/convocatoria_FL_2013_v2.pdf 
Sistema de Información Agroalimentaria y Pesquera (SIAP). (2019). Anuario estadístico de la producción ganadera. Recuperado de https://nube.siap.gob. $\mathrm{mx} /$ cierre_pecuario/

Sonnino, A., y Ruane, J. (2013). La innovación en agricultura como herramientas de la política de seguridad alimentaria: el caso de las biotecnologías agrícolas. En E. Hodson y T. Zamudio (eds.), Biotecnologías e innovación: el compromiso social de la ciencia (pp. 25-52). Bogotá, D C.: Pontificia Universidad Javeriana. Recuperado de http: //www.fao.org/3/ar635s/ar635s.pdf

Sørensen, E., y Torfing, J. (2016). Metagoverning collaborative innovation in governance networks. The American Review of Public Administration, 47(7), 826-839. doi: https://doi.org/10.1177/0275074016643181

Torfing, J., y Triantafillou, P. (2016). Enhancing Public Innovation by Transforming Public Governance? En Autores (eds.), Enhancing Public Innovation by Transforming Public Governance (pp. 1-32), Cambridge: Cambridge University Press. doi: https://doi.org/10.1017/CB09781316105337.001

Torres, G., y Sanz, J. (2007). Construcción de capital social y sistemas agroalimentarios locales en áreas peri-urbanas de la ciudad de México. En R. Méndez (coord.), Economías, mercados de trabajo y territorios metropolitanos en transformación (pp. 573-602). Madrid: Arethuse.

Vázquez-Barquero, A. (2018). Constitución, desarrollo endógeno y dinámica de las instituciones. Revista de Economía Mundial (48), 201-220. Recuperado de http://www.conectadel.org/wp-content/ uploads / 2018/04/2018-AVB-CONSTITUCION-Y-DESARROLLO-ENDOGENO-REM-48.pdf 